\title{
Ligand-field symmetry effects in Fe(II) polypyridyl compounds probed by transient $\mathrm{X}$-ray absorption spectroscopy $\dagger$
}

\author{
Hana Cho,${ }^{a b}$ Matthew L. Strader, ${ }^{a}$ Kiryong Hong, ${ }^{b}$ Lindsey Jamula, ${ }^{c}$ \\ Eric M. Gullikson, ${ }^{d}$ Tae Kyu Kim, ${ }^{* b}$ Frank M. F. de Groot, ${ }^{e}$ \\ James K. McCusker, ${ }^{c}$ Robert W. Schoenlein ${ }^{a}$ and Nils Huse ${ }^{* a f}$
}

\author{
Received 28th February 2012, Accepted 11th May 2012 \\ DOI: $10.1039 / \mathrm{c} 2 \mathrm{fd} 20040 \mathrm{f}$
}

\begin{abstract}
Ultrafast excited-state evolution in polypyridyl Fe(II) complexes is of fundamental interest for understanding the origins of the sub-ps spin-state changes that occur upon photoexcitation of this class of compounds as well as for the potential impact such ultrafast dynamics have on incorporation of these compounds in solar energy conversion schemes or switchable optical storage technologies. We have demonstrated that ground-state and, more importantly, ultrafast time-resolved X-ray absorption methods can offer unique insights into the interplay between electronic and geometric structure that underpins the photo-induced dynamics of this class of compounds. The present contribution examines in greater detail how the symmetry of the ligand field surrounding the metal ion can be probed using these X-ray techniques. In particular, we show that steady-state K-edge spectroscopy of the nearest-neighbour nitrogen atoms reveals the characteristic chemical environment of the respective ligands and suggests an interesting target for future charge-transfer femtosecond and attosecond spectroscopy in the X-ray water window.
\end{abstract}

\section{Introduction}

Transition metal-based polypyridyl complexes represent a large and important class of inorganic compounds. Historically, compounds of $\mathrm{Ru}(\mathrm{II}), \mathrm{Os}(\mathrm{II})$, and $\mathrm{Re}$ (I) have garnered most of the attention from researchers. However, potential limitations of such systems in more applied contexts due to the intrinsic scarcity of these elements has refocused attention on complexes based on the more earth-abundant members of the first transition series; of these, $\mathrm{Fe}(\mathrm{II})$-based chromophores are among the most widely studied in terms of their photophysical properties. In particular, polypyridyl $\mathrm{Fe}^{\mathrm{II}}$ complexes are considered as potential candidates for dye-sensitized solar cells. ${ }^{1,2}$ Initially, photo-perturbation and laser temperature jump measurements on

\footnotetext{
${ }^{a}$ Ultrafast X-ray Science Laboratory, Chemical Sciences Division, Lawrence Berkeley National Laboratory, Berkeley, California, USA

${ }^{b}$ Department of Chemistry and Chemistry Institute of Functional Materials, Pusan National University,Busan, Republic of Korea.E-mail: tkkim@pusan.ac.kr

'Department of Chemistry, Michigan State University, East Lansing, Michigan, USA

${ }^{d}$ Center for X-Ray Optics, Lawrence Berkeley National Laboratory, Berkeley, California, USA

${ }^{e}$ Department of Chemistry, Utrecht University, Utrecht, Netherlands

${ }^{f}$ Max Planck Research Department for Structural Dynamics at the University of Hamburg \& Center for Free Electron Laser Science, Hamburg, Germany. E-mail: nils.huse@mpsd.cfel.de

$\dagger$ Electronic supplementary information (ESI) available. See DOI: 10.1039/c2fd20040f
} 
polypyridyl $\mathrm{Fe}^{\mathrm{II}}$ spin-crossover (SCO) complexes with a low-spin (LS) ${ }^{1}$ Aiegratude Online state were used to characterize the transient high-spin (HS) ${ }^{5} \mathrm{~T}_{2}$ state and to determine the intramolecular kinetics of ground-state recovery, i.e., the ${ }^{5} \mathrm{~T}_{2} \rightarrow{ }^{1} \mathrm{~A}_{1}$ relaxation process., ${ }^{3,4}$ This HS $\rightarrow$ LS relaxation can generally be described in terms of a thermally activated non-adiabatic multi-phonon process in the strong coupling limit. ${ }^{5}$ At sufficiently low temperatures, deactivation of these promoting modes results in light-induced excited spin-state trapping (LIESST) ${ }^{6-8}$ with tunnelling rates for the high spin-to-low spin conversion process as low as $10^{-6} \mathrm{~s}^{-1}$, a phenomenon that has generated considerable interest in potential applications for these molecules in storage devices. ${ }^{9}$

Ultrafast electronic absorption measurements revealed the sub-picosecond dynamics associated with formation of the ${ }^{5} \mathrm{~T}_{2}$ state following photo-excitation of low-spin $\mathrm{Fe}$ (II) complexes ${ }^{10,11}$ as well as a sub-100 fs time scale of charge transferto-ligand field-state conversion. ${ }^{2} \mathrm{~A}$ definitive combined electronic/resonance Raman study detailed a $c a .200 \mathrm{fs}$ time scale for establishment of the ${ }^{5} \mathrm{~T}_{2}$ transient electronic state. ${ }^{12} \mathrm{~A}$ transient infrared spectroscopic study has reported formations times of the ${ }^{5} \mathrm{~T}_{2}$ state and subsequent intramolecular energy redistribution consistent with these findings. ${ }^{13}$ Ultrafast optical studies by Chergui and co-workers provided important additional mechanistic information on the ultrafast spin-state conversion with evidence for ultrafast ${ }^{1} \mathrm{MLCT} \rightarrow{ }^{3} \mathrm{MLCT}$ before subsequent population of the ${ }^{5} \mathrm{~T}_{2}$ high-spin state. ${ }^{14}$ Interestingly, no signatures of vibrational coherence of the symmetric breathing mode have been observed, although this mode is likely coupled to the LS $\rightarrow$ HS conversion due to the $c a$. $0.2 \AA$ increase in bond length that characterizes the transition. Fast population of the ${ }^{5} \mathrm{~T}_{2}$ high-spin state appears to be impulsive on the time-scale of some vibrational modes of the ${ }^{5} \mathrm{~T}_{2}$ state, ${ }^{15}$ having been assigned as the origin of vibrational coherences observed upon formation of the transient high-spin state. A detailed theoretical description of these dynamics has yet to be reported, however, recent studies by de Graaf and Sousa suggest that ultrafast MLCT $\rightarrow{ }^{5} \mathrm{~T}_{2}$ relaxation may occur due to favourable potential energy surface crossings ${ }^{16,17}$ and Veenendaal's group showed that for certain energy level configurations ultrafast spin-cross over between two states could be mediated by a third state in the presence of vibrational manifolds. ${ }^{18,19}$

Complementary to optical techniques, core-level spectroscopy techniques have provided information on the structural dynamics and the accompanying changes in valence charge distribution underlying the SCO transition. ${ }^{20-26}$ Compared to time-resolved optical spectroscopy, ultrafast X-ray spectroscopy is chemically very specific due to the highly localized initial state of the core-level transition. Well-separated transition energies allow for probing specific atomic species with information on molecular structures and valence electronic configurations in the excited state. ${ }^{27-31}$ Transient extended X-ray absorption fine structure (EXAFS) spectroscopy has been employed to study the structural changes around the metal centre via the Fe $\mathrm{K}$-edge of $\left[\mathrm{Fe}\left(\operatorname{tren}(\mathrm{py})_{3}\right)\right]^{2+}$ and $\left[\mathrm{Fe}(\mathrm{bpy})_{3}\right]^{2+}$, providing information on the geometric changes (e.g., $\sim 0.2 \mathrm{~A}$ dilation of the $\mathrm{Fe}-\mathrm{N}$ bonds) subsequent to optical excitation. ${ }^{20,21}$ Transient X-ray diffraction from solid-state SCO complexes has revealed a thermal SCO transition following the initial photo-induced SCO and thermalization of the optical excitation. ${ }^{32}$ Transient X-ray absorption and emission spectroscopy of first-row transition metals can report on spin-state changes on ultrafast time-scales and can reveal electronic changes associated with valence orbitals of specific symmetry. ${ }^{24-26}$ For instance, we have recently demonstrated picosecond and femtosecond XAS in the soft X-ray range of solvated $\left[\mathrm{Fe}\left(\operatorname{tren}(\mathrm{py})_{3}\right)\right]^{2+}$, which provided a detailed picture of the changes in valence electron distributions in the $3 \mathrm{~d}$ manifold of the transient high-spin state in an $\mathrm{Fe}^{\mathrm{II}}$ complex subsequent to charge-transfer excitation. ${ }^{24,26}$ These results underscore the potential of ultrafast L-edge spectroscopy for the study of transition metal chemistry in solution. Furthermore, recent advances in ab initio modelling of X-ray absorption near-edge structures $(\mathrm{XANES})^{33}$ show very good agreement with experimental $\mathrm{Fe}$ K-edge 

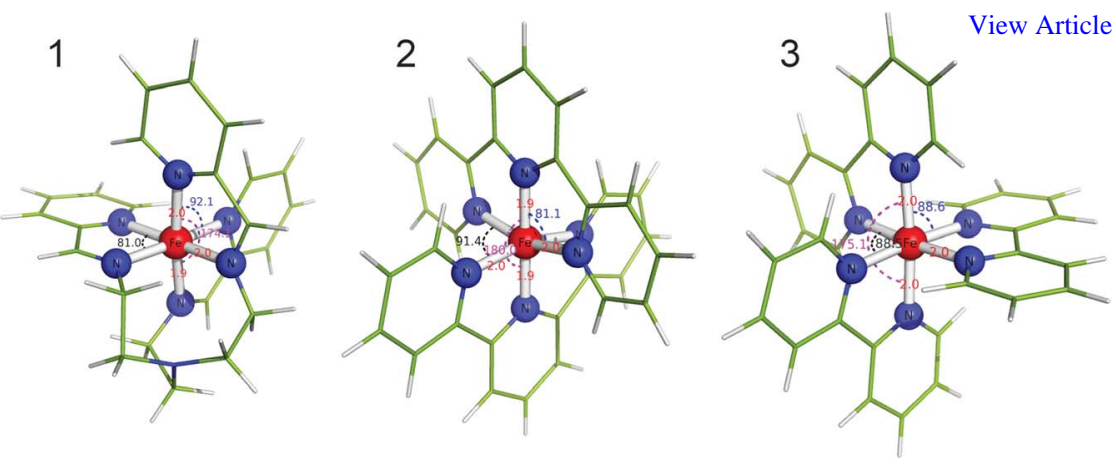

Fig. 1 DFT-derived molecular structures of the investigated polypyridyl Fe ${ }^{\mathrm{II}}$ compounds. 1: $\left[\mathrm{Fe}\left(\operatorname{tren}(\mathrm{py})_{3}\right)\right]^{2+}$. 2: $\left[\mathrm{Fe}(\operatorname{terpy})_{2}\right]^{2+}$. 3: $\left[\mathrm{Fe}(\mathrm{bpy})_{3}\right]^{2+}$. All three compounds feature a pseudo-octahedral arrangement of six nitrogen atoms around the central $\mathrm{Fe}^{\mathrm{II}}$ ion. The deviations from octahedral symmetry vary but are substantial in all three compounds.

spectra, allowing for a more detailed and confident interpretation of XANES spectra in general.

In this work, we aim to understand changes of valence charge density in polypyridyl $\mathrm{Fe}^{\mathrm{II}}$ complexes upon spin-crossover for different, but related, coordination environments of the metal centre. Specifically, we studied the three compounds displayed in Fig. 1 to examine the extent to which steady-state and time-resolved X-ray absorption spectroscopy techniques could provide information concerning small variations in the local ligand field of the $\mathrm{Fe}^{\mathrm{II}}$ ion. The compounds in Fig. 1 are often approximated as possessing $O_{\mathrm{h}}$ symmetry; in reality, the three pyridine and three imine nitrogen donors of compound 1 effectively reduce this to $C_{3}$ symmetry. And while compounds $\mathbf{2}$ and $\mathbf{3}$ all present pyridine nitrogen donors to the metal centre, distortions due to the geometric constraints of the ligands result in symmetry reductions to $C_{2}$ and $D_{3}$ symmetry, respectively. In principle, even a slight reduction of molecular symmetry formally lifts some of the orbital degeneracies, which may in turn noticeably affect electronic interactions between the metal centre and the ligands and give rise to detectable perturbations in the X-ray absorption properties of both the ground- and photo-induced excited states of these compounds.

\section{Methods}

\section{Synthesis}

All three of these $\mathrm{Fe}(\mathrm{II})$ complexes have been previously reported in the literature. $\left[\mathrm{Fe}\left(\operatorname{tren}(\mathrm{py})_{3}\right)\right]\left(\mathrm{PF}_{6}\right)_{2}$ was prepared from $\mathrm{FeCl}_{2} \cdot 4 \mathrm{H}_{2} \mathrm{O}$ reacting with the condensate of three equivalents of 2-pyridine-carboxaldehyde and tris(2-aminoethyl)amine in $\mathrm{MeOH}$ solution under a nitrogen atmosphere as described elsewhere. ${ }^{2}$ Both $[\mathrm{Fe}($ terpy $\left.)_{2}\right]\left(\mathrm{PF}_{6}\right)_{2}$ and $\left[\mathrm{Fe}(\mathrm{bpy})_{3}\right]\left(\mathrm{PF}_{6}\right)_{2}$ were prepared in a similar fashion using $\mathrm{FeCl}_{2} \cdot 4 \mathrm{H}_{2} \mathrm{O}$ and appropriate stoichiometric equivalents of $2,2^{\prime}: 6^{\prime}, 2^{\prime \prime}$-terpyridine and 2,2'-bipyridine, respectively. Sample identities and purities were confirmed using elemental analysis, electrospray mass spectroscopy, and comparison of their optical and electrochemical properties with known samples.

\section{Spectral measurements}

Solution-phase X-ray absorption spectra of the ground state and transient excited state of the samples were measured at the ultrafast soft X-ray beamline of the Advanced Light Source (ALS) while the ground-state spectra of the crystalline compounds were recorded at the EUV calibration beamline of the ALS. Fig. 2 

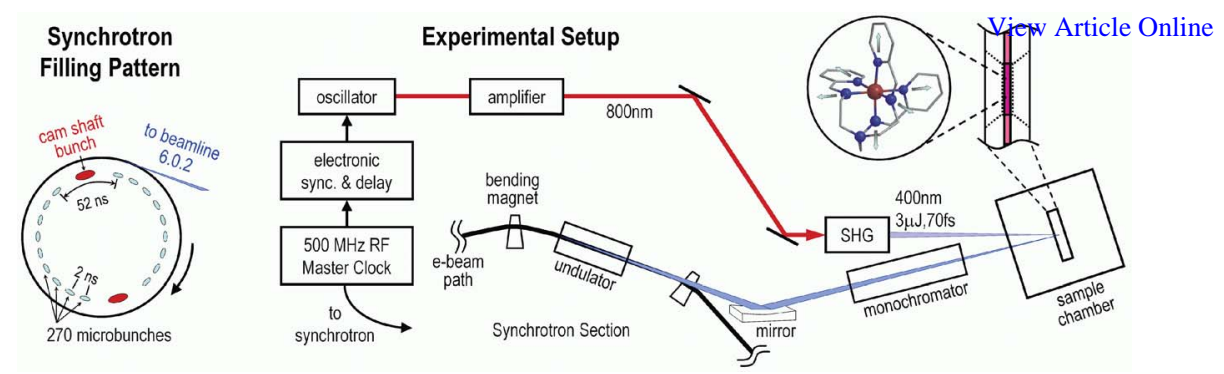

Fig. 2 Schematic of the laser-pump/X-ray-probe experiments at the ultrafast X-ray facility of the Advanced Light Source. The tuneable X-ray pulses produced by the isolated cam shaft electron bunch are focused onto the sample with an imaging monochromator to probe sample transmission changes after laser excitation. The sample is held in a $2 \mu \mathrm{m}$ thin liquid cell inside a soft X-ray compatible experimental chamber.

depicts the general layout of the time-resolved experiment: The ALS synchrotron has a filling pattern with one electron bunch separated from all other bunches by $50 \mathrm{~ns}$ gaps. The $70 \mathrm{ps} \mathrm{X}$-ray pulses generated in the beamline undulator by this so-called cam-shaft bunch are recorded by gated detection. A pulsed Ti:Sapphire laser oscillator with $62.5 \mathrm{MHz}$ repetition rate is synchronized to the $500 \mathrm{MHz}$ radio frequency $(\mathrm{RF})$ with which the electron bunches are driven inside the synchrotron. Appropriately selected and amplified $800 \mathrm{~nm}$ pulses are converted to $400 \mathrm{~nm}$ pulses via second harmonic generation (SHG) and overlapped with the X-rays in the plane of the sample.

\section{Experimental setup}

The details of the sample cell and the experimental chamber have been described elsewhere. ${ }^{24}$ Briefly, a $2 \mu \mathrm{m}$-thick liquid film of a $100 \mathrm{mM}$ acetonitrile solution of polypyridyl $\mathrm{Fe}^{\mathrm{II}}$ complexes was held between two $100 \mathrm{~nm}$-thick silicon nitride membranes and the solid samples were deposited on a thin silicon nitride substrate with a sample thickness of a few hundred nanometres. The liquid samples were excited by $400 \mathrm{~nm}$ laser pulses ${ }^{34}$ with $3 \mu \mathrm{J}$ pulse energy at a repetition rate of $1 \mathrm{kHz}$ and probed with tuneable $\mathrm{X}$-ray pulses at the iron $\mathrm{L}_{2,3}$-edges at around $700 \mathrm{eV}$ by recording changes in the transmitted X-ray intensity at $2 \mathrm{kHz}$ (Fig. 2). Transient differential X-ray absorption spectra at fixed time delay and transients at fixed X-ray photon energy were obtained by taking the ratio of X-ray transmitted intensities of the unexcited and laser-excited samples. ${ }^{30}$

\section{Density functional calculations}

The low-spin ground state of $\left[\mathrm{Fe}(\mathrm{bpy})_{3}\right]^{2+},\left[\mathrm{Fe}(\operatorname{terpy})_{2}\right]^{2+}$, and $\left[\mathrm{Fe}\left(\operatorname{tren}(\mathrm{py})_{3}\right)\right]^{2+}$ in the gas phase were calculated using density functional theory (DFT). ${ }^{35,36}$ All geometries of these complexes were fully optimized using the hybrid functional of Perdew, Burke, and Ernzerhof (PBE0). ${ }^{37}$ We used the effective core potential (ECP) to treat the scalar relativistic effect for Fe. The Los Alamos effective core potential with corresponding valence basis set and polarization $f$ functions, LANL08(f $)^{38,39}$ and 6-31G(d,p) basis sets were used for Fe and the other atoms $(\mathrm{C}, \mathrm{H}$, and $\mathrm{N})$, respectively. To obtain the zero point energy (ZPE), we carried out vibrational frequency calculations by using the same level of theory. We used the natural population analysis (NPA) ${ }^{40}$ for characterization of atomic charges and electronic structures. We also performed the same level of calculations (PBE0/LANL08(f) $+6-31 G(d, p)$ ) for the three $\mathrm{Fe}^{\mathrm{II}}$ complexes in acetonitrile solution. The solvent calculations used the integral equation formalism variant of the polarizable continuum model (IEFPCM). ${ }^{41,42}$ The Gaussian 09 program $^{43}$ was used for all DFT calculations. 


\section{Results and discussion}

We have studied the ground and lowest-energy electronic excited states of the three compounds shown in Fig. 1 by time-resolved X-ray absorption spectroscopy at the iron $\mathrm{L}_{2,3}$-edges. The ground-state electronic absorption spectra of the compounds are plotted in Fig. 3A, along with the probing scheme where electrons are excited from spin-orbit-split Fe-2p core-levels to the unoccupied Fe-3d valence orbitals. This type of core-level spectroscopy can be exploited to extract information on changes of energy levels, valence charge delocalization and bonding, and spin-state changes during the photo-induced intersystem crossing. In particular, the absorption features at the $\mathrm{Fe}_{2,3}$-edges, arising from dipole-allowed resonant $\mathrm{Fe}-2 \mathrm{p}$ transitions, are sensitive to the local electronic structure and dynamic changes in ligand field split $\mathrm{Fe}-3 \mathrm{~d}$ levels. In the following, we present metal (Fe L-edges) and ligand (N K-edge) $\mathrm{X}$-ray absorption spectra, and deduce consequences of structural variation of the ligand cage.

\section{The photo-excited vs. the chemically stabilized high-spin state}

Unlike compound 1 which has a low-spin ${ }^{1} \mathrm{~A}_{1}$ ground state at all temperatures, an analogue in which the protons at the 6-positions of the pyridine rings are replaced with methyl groups exhibits a thermal low-spin to high-spin transition at $\sim 210 \mathrm{~K} .{ }^{44,45}$ For this reason, $\left[\mathrm{Fe}^{\mathrm{II}}(6-\mathrm{Me}-\mathrm{py})_{3}\right.$ tren] $\left(\mathrm{PF}_{6}\right)_{2}$ has served as a roomtemperature high-spin analogue (HSA) in previous studies, allowing for a facile comparison of the HSA ground-state structure and properties with those of the transient high-spin state of the photo-excited compound 1. Previous results from timeresolved visible and EXAFS spectroscopy at the Fe K-edge, ${ }^{2,20}$ clearly illustrated that the structure of the photoexcited high-spin state is very similar to the ground state of $\left[\mathrm{Fe}\left(\operatorname{tren}(6-\mathrm{Me}-\mathrm{py})_{3}\right)\right]^{2+}$, thereby validating its use as a proxy for the transiently formed ${ }^{5} \mathrm{~T}_{2}$ state of the photoexcited low-spin species. The recent ab initio study by van Kuiken and Khalil ${ }^{33}$ also reported substantial structural and electronic resemblance between the transient and chemically stabilized high-spin states. In the following we present experimental results from steady-state and transient X-ray absorption spectroscopy measurements at the $\mathrm{Fe} \mathrm{L}_{2,3}$-edges which complement and extend the information from previous experimental and ab initio results.

Steady-state X-ray absorption spectra of the two solvated compounds at the $\mathrm{Fe}$ $\mathrm{L}_{2,3}$-edges are displayed in Fig. 4A. Shifts to lower energy and distinct spectral reshaping can be observed for the high-spin analogue as compared to the low-spin $\mathrm{L}_{2,3}$-edges of compound 1. The HSA $\mathrm{L}_{3}$-peak is $1.8 \mathrm{eV}$ lower in energy than the
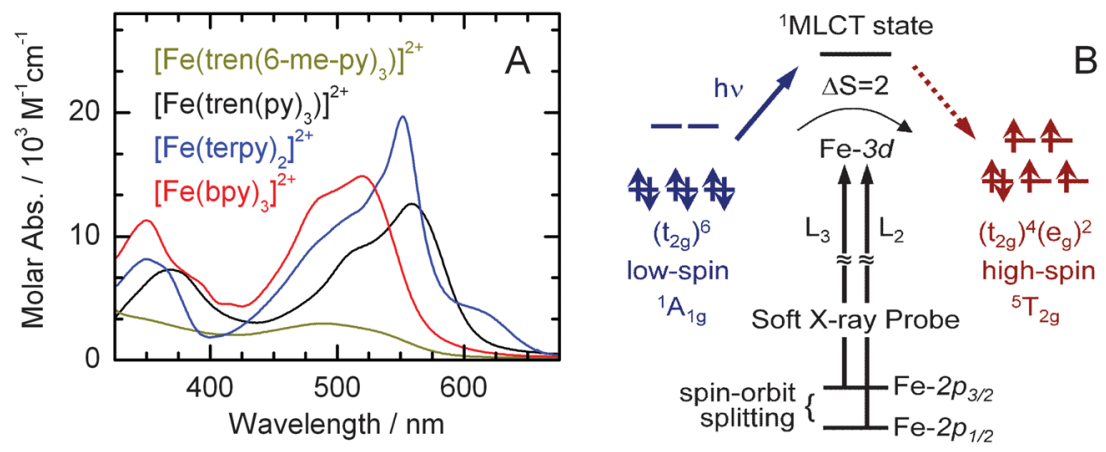

Fig. 3 A Electronic absorption spectra of polypyridyl $\mathrm{Fe}^{\mathrm{II}}$ compounds in acetonitrile solution. B Simplified orbital diagram for an $\mathrm{Fe}^{\mathrm{II}}$ compound in $O_{\mathrm{h}}$ symmetry, illustrating the probing of the low-spin ground state and the transient high-spin excited state via Fe $2 \mathrm{p} \rightarrow 3 \mathrm{~d}$ dipole-allowed core-level transition. 


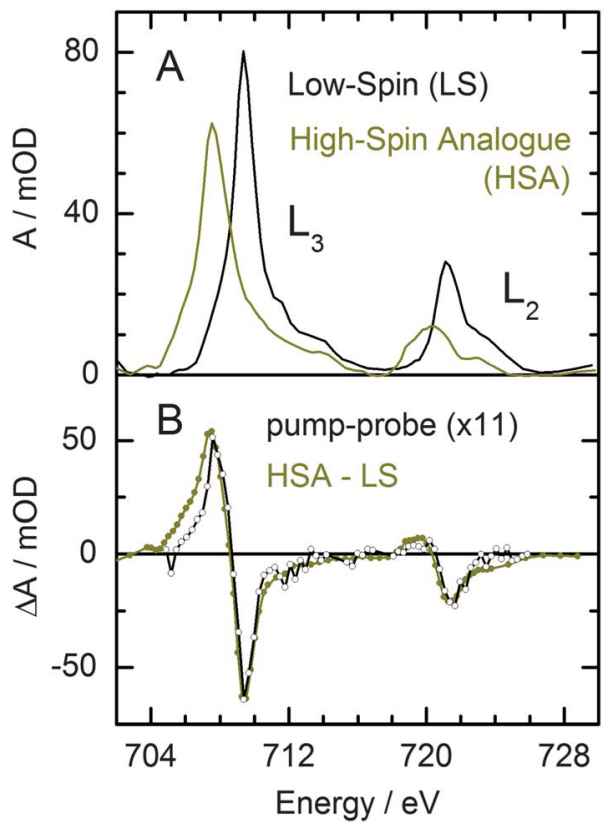

Fig. 4 A Steady-state L-edge spectra of low-spin (LS) $\left[\mathrm{Fe}\left(\operatorname{tren}(\mathrm{py})_{3}\right)\right]^{2+}$ and its high-spin analogue (HSA), both in acetonitrile solution. B A comparison of the calculated difference spectrum of the steady-state L-edge spectra from panel A (HSA-LS, mustard-coloured line) and the experimental transient difference spectrum (black line) of the photo-excited highspin and the ground-state low-spin spectrum recorded $150 \mathrm{ps}$ after excitation of the sample at $400 \mathrm{~nm}$.

LS $\mathrm{L}_{3}$-peak and significantly broader while the centre of the HSA $\mathrm{L}_{2}$-peak is shifted to lower energy by $<1 \mathrm{eV}$ with substantially less intensity than the $\mathrm{LS} \mathrm{L}_{2}$-peak. Great care was taken in the energy calibration of the beamline monochromator to ensure a precision of $0.1 \mathrm{eV}$ during consecutive measurements of the LS and HSA spectra.

Next, we compare the steady-state difference spectrum derived from the data in Fig. 4A with the pump-probe spectrum in Fig. 4B. The rescaling factor of the pump-probe spectrum reflects the fraction of excited molecules $x_{\mathrm{exc}}=1 / 11$ which matches well with our estimates based on $400 \mathrm{~nm}$ excitation fluence and the molar extinction coefficient. The similarity of the two difference spectra is quite striking. All features are reproduced within the signal-to-noise ratio. The most pronounced difference exists at the $\mathrm{L}_{3}$-edge. A larger absorption increase of the dispersive HSA-LS spectrum below $707.6 \mathrm{eV}$ indicates that the red-shift of the HSA $\mathrm{L}_{3}$-edge from that of the LS spectrum is slightly larger than the red-shift of the transient $\mathrm{HS} \mathrm{L}_{3}$-edge. We note that this observation is independent of the uncertainties in the relative absorption of LS and HSA spectra (due to variations in sample concentration and sample thickness) as varying the magnitude of the HSA absorption within experimentally reasonable boundaries shows. Only the maximum absorption loss and gain are affected, not their energetic position.

To understand these spectra we will briefly review our previous work: ${ }^{24} \mathrm{We}$ concluded from comparison of the $\mathrm{L}_{2}$ and $\mathrm{L}_{3}$ absorption line shapes with model systems and multiplet calculations that in the low-spin ${ }^{1} \mathrm{~A}_{1}$ ground state, strong $\pi$-back-bonding results in delocalization of the $\mathrm{Fe} 3 \mathrm{~d}\left(\mathrm{t}_{2 \mathrm{~g}}\right)$ electron into the $\pi^{*}$ orbitals of the tren(py) $)_{3}$ ligands. The high-spin ${ }^{5} \mathrm{~T}_{2}$ excited state is characterized by suppressed $\pi$-back-bonding and attenuated $\sigma$-donation from the tren $(\text { py })_{3}$ ligand relative to the low-spin ground state. Both effects lead to more localized N-2p and $\mathrm{Fe}-3 \mathrm{~d}$ orbitals while keeping the integrated occupancy of the metal-based $\mathrm{e}_{\mathrm{g}}$ and 
$\mathrm{t}_{2 \mathrm{~g}}$ orbitals roughly constant, that is, the overall Fe-3d charge density is unchande Online while the character of the metal-ligand bonding becomes more ionic in nature. These findings show some agreement with a recent $a b$ initio study of the Khalil group. ${ }^{33}$ From the similarity of the difference spectra in Fig. 4B it is apparent that the HSA ground state is very similar to the transient HS state of compound $\mathbf{1}$.

The slightly larger red shift of the high-spin analogue's $\mathrm{L}_{3}$ peak by $\sim 0.1 \mathrm{eV}$ is a manifestation of the reported small differences in structure ${ }^{20}$ and calculated valence charge density ${ }^{33}$ between the transient HS state and the steady-state HSA ground state; the latter exhibits slightly larger bond distances and increased structural distortion compared to octahedral symmetry. Origins of the red shift of the transient HS spectrum are the reduction in energy splitting of the $F e t_{2 g}$ and $e_{g}$ orbitals with increasing bond lengths and increased charge localization. ${ }^{24}$ Hence, the spectral difference in Fig. 4B is experimental evidence of the predicted electronic structure of the HSA.

The $\mathrm{L}_{2}$-edges of transient $\mathrm{HS}$ and HSA are essentially identical. The larger absorption increase at the HSA $\mathrm{L}_{3}$-peak signals a redistribution of absorption from the $\mathrm{L}_{2}$ to $\mathrm{L}_{3}$-edge which is commonly a sign of a higher spin-state or spin admixture but the spin-state has been reported as pure. We think that this behaviour is a result of the larger deviation from octahedral symmetry of the HSA ground state structure compared to the transient HS state as predicted by van Kuiken and Khalil. ${ }^{33}$ We will discuss changes in the relative $\mathrm{L}_{2}$ and $\mathrm{L}_{3}$ intensity in the context of ligand variations in the next section.

\section{Effects of octahedral distortion}

The ligand field in which a transition metal ion is placed has a profound influence on virtually all of the physical properties of the molecule that relate to electronic structure including geometry, optical and magnetic properties, and chemical reactivity. In this context, the question arises what effects ligand variation has on the transient HS state in polypyridyl $\mathrm{Fe}^{\mathrm{II}}$ complexes, and if metal L-edge spectroscopy can detect meaningful differences between the different compounds. The three $\mathrm{Fe}^{\mathrm{II}}$ polypyridyl complexes we are examining provide a convenient platform on which to begin examining these issues given their similar, but nevertheless distinct, symmetry and compositional characteristics.

Ground state spectra of the three $\mathrm{Fe}^{\mathrm{II}}$ complexes are shown in Fig. 5A. Absolute absorption differences are not identical due to variation in solubility and sample thickness on the sub-micron scale; after correcting for sample concentration the peak absorption exhibited variances of only $\sim 10 \%$. Accordingly, the groundstate absorption spectra in Fig. 5 have been normalized to the $\mathrm{L}_{3}$-peak of [Fe(tren$\left.(\text { py) })_{3}\right)_{]^{2+}}$ at $709.4 \mathrm{eV}$ for comparative purposes (black line). The overall spectral shapes are very similar. Compound $\mathbf{1}$ displays a more distinct shoulder on the low-energy side of the $\mathrm{L}_{3}$-lineshape (Fig. 5B) which is weaker in compounds 2 and 3. Given that compound $\mathbf{1}$ is distinct in possessing two different types of nitrogen donors (pyridine- and imine-based), we believe that this difference is a direct reflection of this compositional variation. Conversely, compound $\mathbf{3}$ displays the narrowest absorption features which we attribute to the fact that this molecule possesses the highest point-group symmetry of the three compounds studied. Compounds 1 and 2 show identical peak positions within the spectral precision of $\sim 0.1 \mathrm{eV}$. In contrast, compound 3 is significantly shifted to lower energy by $\sim 0.2 \mathrm{eV}$. This is somewhat surprising as one would expect that the ligand systems of compounds $\mathbf{2}$ and $\mathbf{3}$ with only pyridine groups have very similar spectral positions.

The branching ratio of the integral $\mathrm{L}_{2,3}$-intensities due to $\mathrm{Fe} 2 \mathrm{p} \rightarrow 3 \mathrm{~d}$ transitions as defined by Thole and co-workers, ${ }^{46} r_{3}=I\left(\mathrm{~L}_{3}\right) /\left[I\left(\mathrm{~L}_{2}\right)+I\left(\mathrm{~L}_{3}\right)\right]$, reports on the spinstate, electrostatic interactions between the core-hole and the valence charge density, and the spin-orbit (SO) interactions between the core-hole and the metal$3 \mathrm{~d}$ manifold as well as within the latter. ${ }^{46}$ To properly account for the bound-bound 

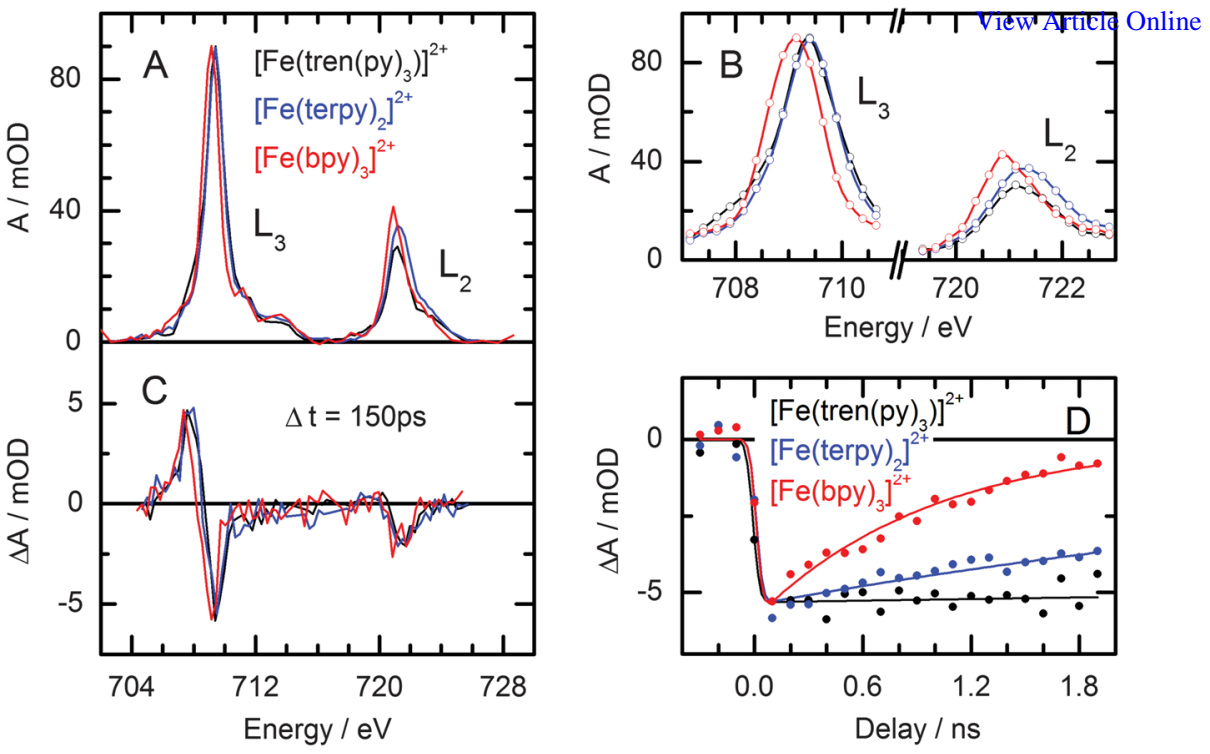

Fig. 5 Comparison of $\left[\mathrm{Fe}\left(\operatorname{tren}(\mathrm{py})_{3}\right)\right]^{2+},\left[\mathrm{Fe}(\text { terpy })_{2}\right]^{2+}$, and $\left[\mathrm{Fe}(\mathrm{bpy})_{3}\right]^{2+}$ in $100 \mathrm{mM}$ acetonitrile solution A Steady-state X-ray absorption spectra at the $\mathrm{Fe}_{2,3}$-edges. B Expanded view of the absorption peaks at the $\mathrm{Fe} \mathrm{L}_{2,3}$-edges (colour coding as in panel A) C Corresponding transient differential X-ray absorption spectra at $150 \mathrm{ps}$ pump-probe delay after excitation at $400 \mathrm{~nm}$. D Pump-probe delay scans at $709.4 \mathrm{eV}$ (dots) and fits of the data to mono-exponential decay models using previously published excited-state lifetimes. See text for further details.

core-level transitions, we have subtracted the continuum edges which are modelled by two arctangents at the $\mathrm{L}_{2,3}$-edges with a lifetime broadening of $0.4 \mathrm{eV}$ and $0.2 \mathrm{eV}$, respectively. Some residual absorption at around $713 \mathrm{eV}$ stems from a Gaussian-like feature in the EXAFS spectrum of the $\mathrm{PF}_{6}{ }^{-}$fluorine K-edge. It accounts for $8 \%$ of the $\mathrm{L}_{3}$-edge intensity and we have corrected the branching ratio accordingly. The branching ratio $r_{3}$ equals 0.67 for both the pyridine compounds 2 and $\mathbf{3}$ which contain terpy and bpy ligands, respectively, while it amounts to 0.71 in compound 1 containing the tren(py) $)_{3}$ ligand. For compounds with singlet ground states, the branching ratio is typically $\sim 0.6$ while in the absence of electrostatic and SO interactions, the branching ratio is statistical, amounting to $2 / 3$. We conclude from empirical charge-transfer multiplet calculations (see supplementary material for details $\dagger$ ) that the chemical differences in the ligand cage between compound $\mathbf{1}$ on one hand and compounds $\mathbf{2}$ and $\mathbf{3}$ on the other lead to variations in electrostatic and SO interactions that manifest to a measurable extent in the branching ratio of the groundstate Fe-2p spectra as observed in Fig. 5A,B. Ab initio multiplet calculations would be very beneficial to gain more detailed insight into the two interactions discussed. While currently no such program code exists, recent developments may soon provide $a b$ initio descriptions of third-row transition metal $2 \mathrm{p}$ spectra. ${ }^{47}$

Fig. 5C shows the normalized changes upon $400 \mathrm{~nm}$ excitation at a time delay of 150 ps. The spectral features are identical within the signal-to-noise ratio ( $\sim 0.5$ mOD r.m.s.) of the experiment. This empirical observation implies that the differences in geometry noted in the ground states of these compounds are reflected in their respective high-spin excited states, as well.

Fig. 5D shows pump-probe delay scans at a fixed probe energy of $709.4 \mathrm{eV}$, the region of maximal ground state bleaching. These transients are probing groundstate recovery dynamics, i.e., the ${ }^{5} \mathrm{~T}_{2} \rightarrow{ }^{1} \mathrm{~A}_{1}$ relaxation process subsequent to photo-induced formation of the transient high-spin state. The differences in lifetimes inferred from these data are readily understandable within the context of 
non-radiative decay theory and known variations in high-spin/low-spin energies across this series; this has been discussed at length in the literature (see Hauser et al. ${ }^{48}$ and references therein) and will not be explored further here. We briefly list the published room-temperature ${ }^{5} \mathrm{~T}_{2}$ lifetimes of the three compounds in acetonitrile solution. 1: $\tau_{\mathrm{HS}}=60 \pm 5 \mathrm{~ns}, 2: \tau_{\mathrm{HS}}=5.4 \pm 0.1 \mathrm{~ns}$, and 3: $\tau_{\mathrm{HS}}=$ $980 \pm 50$ ps. It can be seen that the data in Fig. 5D can be considered wholly consistent with the corresponding optical data over this temporal range, indicating that these X-ray measurements are reliable probes of ground-state recovery, as well.

\section{Chemical signatures of nearest-neighbour nitrogen atoms}

An additional approach to more detailed information concerning metal-ligand interactions in coordination compounds is to focus on the atoms bound to the metal centre(s). These lighter elements such as carbon, nitrogen, and oxygen can reveal the 'ligand perspective' by probing their $1 \mathrm{~s} \rightarrow 2 \mathrm{p}$ (and higher energy) core-level transitions. The former transition probes the LUMO of the metal centre's nearest neighbours while core-level excitation at higher energy reveal spectrally broader continuum resonances which can also be sensitive to the chemical environment and changes thereof. ${ }^{49}$ In the following we present data on crystalline films of $\mathrm{PF}_{6}$-salts of the three compounds in Fig. 1 that we recorded at the Advanced Light Source' EUV calibration beamline 6.3.2 to explore the potential of nitrogen K-edge spectroscopy to elucidate the local chemical environment.

Fig. 6 shows the nitrogen K-edge absorption spectra of compounds 1, 2, and 3 around $400 \mathrm{eV}$. The left panel A features the $1 \mathrm{~s} \rightarrow 2 \mathrm{p}$. The bipyridine and terpyridine ligands have identical $1 \mathrm{~s} \rightarrow 2 \mathrm{p}$ transitions at $399.5 \mathrm{eV}$ with additional absorption structure characteristic of each ligand. While these two ligand types feature only pyridine ligands, the tren $(\mathrm{py})_{3}$ ligand cage contains two types of nearest-neighbour nitrogen atoms (those from the pyridine and the imine groups) which lead to two unresolved transitions centred at $399.2 \mathrm{eV}$, resulting in a seemingly broader lineshape that reflects the two chemically distinct nitrogen species. These observations are commensurate with the observations of differing branching ratios of compound $\mathbf{1}$ versus the purely pyridine-based compounds $\mathbf{2}$ and $\mathbf{3}$. However, the latter two compounds show distinct differences in the $\mathrm{N} \mathrm{K}$-edge spectra above $400 \mathrm{eV}$ which makes this type of XANES spectroscopy an interesting and chemically specific target.

The same spectra are plotted in Fig. 6B over a wider energy range. In all three spectra a broader absorption feature that starts to rise at $403 \mathrm{eV}$ at energies above the core level ionization threshold as a continuum resonance can be found. The latter may be interpreted as shape resonances, i.e. they can be thought of as unoccupied
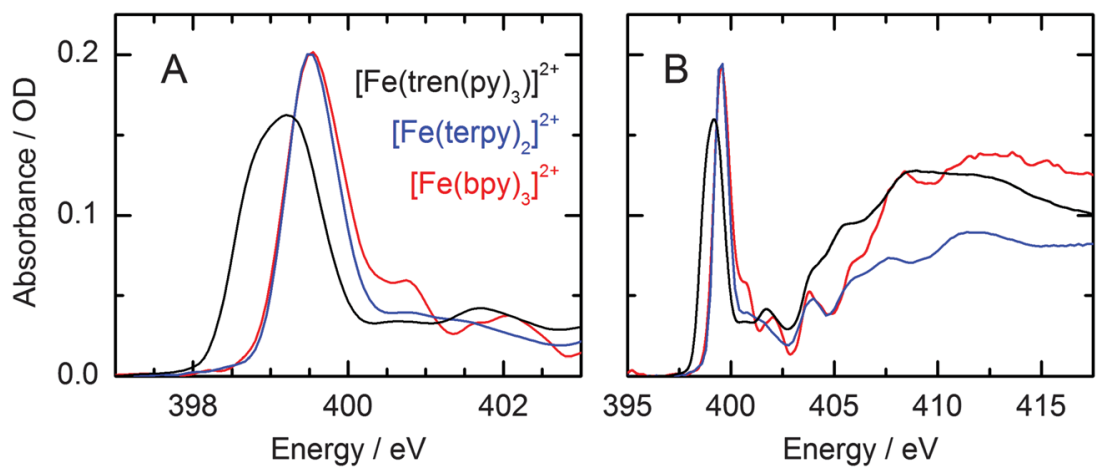

Fig. 6 Ground-state nitrogen K-edge spectra of thin solid films of $\left[\mathrm{Fe}\left(\operatorname{tren}(\mathrm{py})_{3}\right)\right]\left(\mathrm{PF}_{6}\right)_{2}$, $\left[\mathrm{Fe}(\text { terpy })_{2}\right]\left(\mathrm{PF}_{6}\right)_{2}$, and $\left[\mathrm{Fe}(\mathrm{bpy})_{3}\right]\left(\mathrm{PF}_{6}\right)_{2}$. A Nitrogen $1 \mathrm{~s} \rightarrow 2 \mathrm{p}$ transitions. B Broader spectral energy range illustrating both Nitrogen $1 \mathrm{~s} \rightarrow 2 \mathrm{p}$ and $\sigma^{*}$ shape resonances. 
molecular orbitals embedded in the continuum to which the core-excited promoted. However, there is still some dispute over the nature of these absorption features. ${ }^{50}$ Generally, transient nitrogen K-edge edge spectroscopy could prove useful in clarifying whether these resonances provide a measure of bond length changes during chemical reactions, e.g. shifting to higher energy with decreasing chemical bonds. More specifically, this type of spectroscopy on solvated transition-metal complexes could prove very useful in understanding additional details of the metal-ligand interactions. For instance, to address the question of why these complexes, when excited to the MLCT manifold relax to the high-spin ${ }^{5} \mathrm{~T}_{2}$ state with time-constants of $\sim 100$ fs without a clear signature of involving metal-centred ligand-field states that are energetically intermediate between the MLCT manifold and the high-spin ${ }^{5} \mathrm{~T}_{2}$ state. One advantage of $\mathrm{K}$-edge spectroscopy in general has been that the core-excited electron can be treated in a one-electron picture which allows for ab initio methods to simulate core-level spectra (in contrast to L-edge spectra of first-row transition metals in which multiplets due to strong spin-orbit interactions have complicated the development of ab initio methods for core-level spectroscopy. For transition metals heavier than those of the first row, multiplet effects are relatively weak allowing for reliable $a b$ initio-based simulations of transient L-edge spectra in analogous molecular systems ${ }^{51}$ ). Ab initio studies of 1 s transitions of light elements would provide direct comparison with experiment and make details of the evolving valence charge density of the nearest neighbour atoms in transition-metal complexes accessible. Last we note that rapid progress in laser-based high-harmonic sources has led to laser technology with photon energies in the soft X-ray regime. ${ }^{52}$ It can be anticipated that in the next years laser-based ultrashort $\mathrm{X}$-ray sources $(<30 \mathrm{fs}$ ) with sufficient flux at absorption edges in the lower soft X-ray range will allow for femtosecond and possibly attosecond spectroscopy in solution to study charge migration and atomic rearrangement on their natural time scales.

\section{Conclusions}

We have studied polypyridyl $\mathrm{Fe}^{\mathrm{II}}$ complexes in solution with emphasis on the effects of ligand variation on the valence charge density using time-resolved core-level spectroscopy at the $\mathrm{Fe}_{2,3}$-edges. In particular, the transiently excited high-spin state of $\left[\mathrm{Fe}\left(\operatorname{tren}(\mathrm{py})_{3}\right)\right]^{2+}$ and its high-spin analogue have very similar Fe-2p spectra which points to the great similarity of their respective valence charge densities, complementing previous experimental findings ${ }^{2,20}$ and supporting detailed predictions from recent $a b$ initio calculations..$^{33}$ We further conclude from comparing groundstate and transient iron L-edge spectra of three similar polypyridyl $\mathrm{Fe}^{\mathrm{II}}$ complexes that the metal valence charge densities in these compounds are similar. However, varying chemical composition and ligand-field symmetry influence the valence charge densities of ground and excited states as probed by transient L-edge spectroscopy. Symmetry effects could be an important factor in influencing the dynamics that lead to high-spin state formation. Recent theoretical work by van Veenendaal's group ${ }^{18,19}$ proposes fast high-spin formation upon MLCT excitation due to energetic proximity of a third state which mediates ultrafast spin-cross over via spin-orbit interactions. De Graaf and Sousa relate to this work, suggesting that deviations from octahedral symmetry might by relevant in this context. ${ }^{17}$ Hence, systematic ligand variation in conjunction with ultrafast L-edge spectroscopy could shed more light on ligand-field symmetry. Additionally, ultrafast spectroscopy at the K-edges of the nearest-neighbours of metal centres in solvated transition-metal compounds is proposed to probe the 'ligand view' of the metal-ligand interactions. Such measurements will provide complementary information on the valence charge density of short-lived intermediates in combination with newly developed $a b$ initio methods for core-level spectroscopy. We anticipate that advances in laser technology will allow for 'table-top' experiments in the foreseeable future with time-resolution approaching the fundamental time-scales of charge transfer. Such techniques could 
shed further light on elementary chemical processes beyond the prototypicalAititine Online complexes studied in this work.

\section{Acknowledgements}

This work was supported by the Director, Office of Science, Office of Basic Energy Sciences, the Chemical Sciences, Geosciences, and Biosciences Division under the Department of Energy, Contract No. DE-AC02-05CH11231 (N.H., H.C., and R.W.S.) and Grant No. DE-FG02-01ER15282 (J.K.M.), as well as the National Research Foundation of Korea (NRF) funded by the Korea government (Grant No. 2009-0068446, 2010-0006570, and 2007-0056330), MEST, PAL, and the Korean XFEL project (H. C., K. H., and T.K.K.).

\section{References}

1 S. Ferrere and B. A. Gregg, J. Am. Chem. Soc., 1998, 120, 843.

2 J. E. Monat and J. K. McCusker, J. Am. Chem. Soc., 2000, 122, 4092.

3 C. Creutz, M. Chou, T. L. Netzel, M. Okumura and N. Sutin, J. Am. Chem. Soc., 1980, 102, 1309.

4 J. J. McGravey and I. Lawthers, J. Chem. Soc., Chem. Commun., 1982, 902.

5 E. Buhks, G. Navon, M. Bixon and J. Jortner, J. Am. Chem. Soc., 1980, 102, 2918.

6 S. Decurtins, P. Gütlich, C. Köhler, H. Spiering and A. Hauser, Chem. Phys. Lett., 1984, $105,1$.

7 C. L. Xie and D. N. Hendrickson, J. Am. Chem. Soc., 1987, 109, 6981.

8 P. Gütlich and A. Hauser, Coord. Chem. Rev., 1990, 97, 1.

9 A. Hauser, A. Vef and P. Adler, J. Chem. Phys., 1991, 95, 8710.

10 J. K. McCusker, K. N. Walda, R. C. Dunn, J. D. Simon, D. Magde and D. N. Hendrickson, J. Am. Chem. Soc., 1992, 114, 6919.

11 J. K. McCusker, K. N. Walda, R. C. Dunn, J. D. Simon, D. Magde and D. N. Hendrickson, J. Am. Chem. Soc., 1993, 115, 298.

12 A. L. Smeigh, M. Creelman, R. A. Mathies and J. K. McCusker, J. Am. Chem. Soc., 2008, 130, 14105.

13 M. M. N. Wolf, R. Groß, C. Schumann, J. A. Wolny, V. Schünemann, A. D, H. Paulsen, J. J. McGarvey and R. Diller, Phys. Chem. Chem. Phys., 2008, 10, 4264.

14 W. Gawelda, A. Cannizzo, V.-T. Pham, F. van Mourik, C. Bressler and M. Chergui, J. Am. Chem. Soc., 2007, 129, 8199.

15 C. Consani, M. Prémont-Schwarz, A. ElNahhas, C. Bressler, F. van Mourik, A. Cannizzo and M. Chergui, Angew. Chem., Int. Ed., 2009, 48, 7184.

16 C. de Graaf and C. Sousa, Chem.-Eur. J., 2010, 16, 4550.

17 C. de Graaf and C. Sousa, Int. J. Quantum Chem., 2011, 111, 3385.

18 M. van Veenendaal, J. Chang and A. J. Fedro, Phys. Rev. Lett., 2010, 104, 067401.

19 J. Chang, A. J. Fedro and M. van Veenendaal, Phys. Rev. B, 2010, 82, 075124.

20 M. Khalil, M. A. Marcus, A. L. Smeigh, J. K. McCusker, H. H. W. Chong and R. W. Schoenlein, J. Phys. Chem. A, 2006, 110, 38.

21 W. Gawelda, V.-T. Pham, M. Benfatto, Y. Zaushitsyn, M. Kaiser, D. Grolimund, S. L. Johnson, R. Abela, A. Hauser, C. Bressler and M. Chergui, Phys. Rev. Lett., 2007, 98, 057401.

22 C. Bressler, C. Milne, V.-T. Pham, A. ElNahhas, R. M. van der Veen, W. Gawelda, S. Johnson, P. Beaud, D. Grolimund, M. Kaiser, C. N. Borca, G. Ingold, R. Abela and M. Chergui, Science, 2009, 323, 489.

23 S. Nozawa, T. Sato, M. Chollet, K. Ichiyanagi, A. Tomita, H. Fujii, S.-i. Adachi and S.-y. Koshihara, J. Am. Chem. Soc., 2010, 132, 61.

24 N. Huse, T. K. Kim, L. Jamula, J. K. McCusker, F. M. de Groot and R. W. Schoenlein, J. Am. Chem. Soc., 2010, 132, 6809.

25 G. Vankó, P. Glatzel, V.-T. Pham, R. Abela, D. Grolimund, C. N. Borca, S. L. Johnson, C. J. Milne and C. Bressler, Angew. Chem., Int. Ed., 2010, 49, 1.

26 N. Huse, H. Cho, K. Hong, L. Jamula, F. M. de Groot, T. K. Kim, J. K. McCusker and R. W. Schoenlein, J. Phys. Chem. Lett., 2011, 2, 880.

27 L. X. Chen, W. J. H. Jager, G. Jennings, D. J. Gosztola, A. Munkholm and J. P. Hessler, Science, 2001, 292, 262.

28 C. Bressler and M. Chergui, Chem. Rev., 2004, 104, 1781. 
29 P. Wernet, G. Gavrila, K. Godehusen, C. Weniger, E. Nibbering, T. El Online W. Eberhardt, Appl. Phys. A: Mater. Sci. Process., 2008, 92, 511.

30 N. Huse, H. Wen, D. Nordlund, E. Szilagyi, D. Daranciang, T. A. Miller, A. Nilsson, R. W. Schoenlein and A. M. Lindenberg, Phys. Chem. Chem. Phys., 2009, 11, 3951.

31 N. Huse, H. Wen, R. W. Schoenlein and A. M. Lindenberg, J. Chem. Phys., 2009, 131, 234505 .

32 M. Lorenc, J. Hébert, N. Moisan, E. Trzop, M. Servol, M. Buron-Le Cointe, H. Cailleau, M. Boillot, E. Pontecorvo, M. Wulff, S. Koshihara and E. Collet, Phys. Rev. Lett., 2009, 103, 028301 .

33 B. E. Van Kuiken and M. Khalil, J. Phys. Chem. A, 2011, 115, 10749.

34 Although this wavelength corresponds to excitation into a higher energy absorption feature than we have previously exploited, the sub-picosecond conversion to the high-spin ${ }^{5} \mathrm{~T}_{2}$ state characteristic of these compounds implies that the same electronic excited state is being probed on the 10 s of picosecond time scale relevant for the present study.

35 P. Hohenburg and W. Kohn, Phys. Rev., 1964, 136, B864.

36 W. Kohn and L. J. Sham, Phys. Rev., 140, A113.

37 C. Adamo and V. Barone, J. Chem. Phys., 1999, 110, 6158.

38 P. J. Hay and W. R. Wadt, J. Chem. Phys., 1985, 82, 299.

39 A. Ehlers, M. Böhme, S. Dapprich, A. Gobbi, A. Höllwarth, V. Jonas, K. Köhler, R. Stegmann, A. Veldkamp and G. Frenking, Chem. Phys. Lett., 1993, 208, 111.

40 E. Reed, R. B. Weinstock and F. Weinhold, J. Chem. Phys., 1985, 83, 735.

41 B. Mennucci, E. Cancès and J. Tomasi, J. Phys. Chem. B, 1997, 101, 10506.

42 E. Cancès, B. Mennucci and J. Tomasi, J. Chem. Phys., 1997, 107, 3032.

43 M. J. Frisch, G. W. Trucks, H. B. Schlegel, G. E. Scuseria, M. A. Robb, J. R. Cheeseman, G. Scalmani, V. Barone, B. Mennucci, G. A. Petersson, H. Nakatsuji, M. Caricato, X. Li, H. P. Hratchian, A. F. Izmaylov, J. Bloino, G. Zheng, J. L. Sonnenberg, M. Hada, M. Ehara, K. Toyota, R. Fukuda, J. Hasegawa, M. Ishida, T. Nakajima, Y. Honda, O. Kitao, H. Nakai, T. Vreven, J. A. Montgomery, Jr., J. E. Peralta, F. Ogliaro, M. Bearpark, J. J. Heyd, E. Brothers, K. N. Kudin, V. N. Staroverov, R. Kobayashi, J. Normand, K. Raghavachari, A. Rendell, J. C. Burant, S. S. Iyengar, J. Tomasi, M. Cossi, N. Rega, J. M. Millam, M. Klene, J. E. Knox, J. B. Cross, V. Bakken, C. Adamo, J. Jaramillo, R. Gomperts, R. E. Stratmann, O. Yazyev, A. J. Austin, R. Cammi, C. Pomelli, J. Ochterski, R. L. Martin, K. Morokuma, V. G. Zakrzewski, G. A. Voth, P. Salvador, J. J. Dannenberg, S. Dapprich, A. D. Daniels, O. Farkas, J. B. Foresman, J. V. Ortiz, J. Cioslowski and D. J. Fox, GAUSSIAN 09 (Revision B.01), Gaussian, Inc., Wallingford, CT, 2009.

44 K. M. Kadish, C. H. Su, D. Schaeper, C. L. Merrill and L. J. Wilson, Inorg. Chem., 1982, 21, 3433.

45 A. J. Conti, C. L. Xie and D. N. Hendrickson, J. Am. Chem. Soc., 1989, 111, 1171.

46 B. T. Thole and G. van der Laan, Phys. Rev. B, 1988, 38, 3158.

$47 \mathrm{P}$. Wernet, private communication.

48 A. Hauser, C. Enachescu, M. L. Daku, A. Vargas and N. Amstutz, Coord. Chem. Rev., 2006, 250, 1642.

49 J. M. Garcia-Lastra, P. L. Cook, F. J. Himpsel and A. Rubio, J. Chem. Phys., 2010, 133, 151103 .

50 M. Piancastelli, J. Electron Spectrosc. Relat. Phenom., 1999, 100, 167.

51 B. E. van Kuiken, N. Huse, H. Cho, M. L. Strader, M. S. Lynch, R. W. Schoenlein and M. Khalil, J. Phys. Chem. Lett., 2012, 1695.

52 M.-C. Chen, P. Arpin, T. Popmintchev, M. Gerrity, B. Zhang, M. Seaberg, D. Popmintchev, M. M. Murnane and H. C. Kapteyn, Phys. Rev. Lett., 2010, 105, 173901. 